\title{
WILEY-VCH
}

This is the peer reviewed version of the following article: Advanced Materials 2017, 29, 1700888, which has been published in final form at https://doi.org/10.1002/adma.201700888. This article may be used for non-commercial purposes in accordance with Wiley Terms and Conditions for Use of Self-Archived Versions.

Article type: Communication

\section{Electrostatic Design of 3D Covalent Organic Networks}

Veronika Obersteiner, Andreas Jeindl, Johannes Götz, Aurelie Perveaux, Oliver T. Hofmann, and Egbert Zojer*

Institute of Solid State Physics, NAWI Graz, Graz University of Technology, Petersgasse 16, 8010 Graz, Austria

*egbert.zojer@tugraz.at

Keywords: covalent organic frameworks, electrostatic design, solar cells, density functional theory 


\begin{abstract}
An innovative strategy for electrostatically designing the electronic structure of 3D bulk materials is proposed to control charge carriers at the nanoscale. This is achieved by shifting the electronic levels of chemical identical semiconducting elements through the periodic arrangement of polar functional groups. For the example of covalent organic networks, by first-principles calculations, the resulting collective electrostatic effects are shown to allow a targeted manipulation of their electronic landscape such that spatially confined pathways for electrons and holes can be realized. Mimicking donor-acceptor bulk heterojunctions, the new materials hold high promise for photovoltaic applications. The distinct advantage over the conventional approach of splitting excitons through chemically distinct donor and acceptor units is that here the magnitude of the band offset can be continuously tuned by varying dipole densities. A particularly promising feature of the suggested strategy is its structural versatility, which also enables the realization of more complex quantum structures such as quantum-cascades and quantum-checkerboards.
\end{abstract}


We portray an innovative strategy for designing the electronic structure of $3 \mathrm{D}$ materials, where we use polar functional groups to control the energy landscape and localization of electronic states. The approach is discussed for the prototypical example of bulk heterojunctions. These are commonly applied in organic solar cells as active layers responsible for dissociating the photogenerated excitons. Bulk heterojunctions consist of phase separated electron donating (D) and accepting (A) regions. An approach for achieving a sensitive control over their nano-morphology is incorporating the donating and accepting elements into Covalent-Organic Frameworks (COFs). In this way, D/A bulk heterojunctions with a maximized internal interface can be realized, in which a periodic and well-controlled arrangement of the organic building blocks is ensured by covalent bonds. ${ }^{[1-8]}$ Traditional design strategies for realizing donor-acceptor COFs either use a donor covalently bound to the inner wall of an accepting COF (i.e., wall functionalization) ${ }^{[6]}$ or they rely on constructing the walls from alternatingly donating and accepting functional units. ${ }^{[3,4,7,8]}$ The $\pi$-stacked columns of the layered donor-acceptor sheets then provide pathways for electrons and holes, ${ }^{[1]}$ facilitating charge transport in the stacking direction. The alignment of the electronic states in such type-II heterostructures, i.e. the offsets between the local valence- and conduction-band edges, is usually determined by changing the ionisation energy and electron affinity of the individual donating and accepting segments. ${ }^{[5,6,9,10]}$

In the present contribution, we employ a fundamentally different strategy for controlling the energetic positions and localization of electronic states: We employ chemically identical building blocks as nominally donating and accepting units, but achieve an equivalent band offset as in the D/A systems through so-called collective electrostatic effects. These occur when assembling periodic arrays of polar groups within a material in an ordered fashion. Then the superposition of their electric fields dramatically changes the energy landscape. ${ }^{[1-16]}$ Such an approach has recently been suggested for manipulating the electronic states within graphene $^{[17]}$ and semiconducting self-assembled monolayers (SAMs) on metal substrates. ${ }^{[18]}$ 


\section{WILEY-VCH}

First proof of principle experiments on SAMs have already confirmed the induced potential step within the monolayer ${ }^{[16]}$ and its significant impact on ballistic charge transport through the SAMs. ${ }^{[19]}$

Here we go decisively beyond these approaches, showing how electrostatic design can be used to control charges at a nanoscopic level in bulk materials with the ultimate goal to locally control exciton dissociation and to realize confined pathways for electrons and holes. The distinct advantage over the above-discussed donor-acceptor based bulk heterostructures is that electrostatic design allows a quasi-continuous tuning of the offset between energy levels by varying the dipole density. This can be very useful for solar cells consisting of organic chromophores, as this offset is the driving force for overcoming the often considerable exciton binding energies. At the same time, the level offset needs to be small enough to avoid an excessive loss of energy to the creation of phonons. By a suitable design of the polar sections, electrostatic design also allows the realization of much more complex energy landscapes. As prototypical examples for those, we discuss 3D quantum-cascades and quantum-checkerboards.

The suggested approach is not restricted to a specific class of materials, as it solely relies on the superposition of the electric fields generated by an ordered array of dipolar elements. In fact, it can be applied to any type of structure (be it organic or inorganic) in which regular arrangements of dipolar functional groups can be realized. Therefore, in the present paper we focus on conceptually and structurally simple systems, in which the energetic shifts due to collective electrostatic effects can be seen most clearly without any interference from complex functional elements. Thus we here discuss the electrostatic design idea on the basis of 3D networks consisting of identical semiconducting units (i.e.., chromophore segments). The collective electrostatic effects are realized by a periodic arrangement of linking units containing a dipolar element.

In this way, complex energy landscapes of electronic states can be realized. Prototypical examples of the resulting structures are shown in Figure 1. We choose (zinc)porphyrin units as classical semiconducting chromophores, as they are frequently used in COF-based systems. ${ }^{[20-27]}$ and are also commonly employed in organic solar cells. ${ }^{[28,29]}$ Depending on the specific position of the porphyrin in the structure and the linking direction, they are connected either by triacetylene groups (apolar and chosen to avoid any steric complications) or by linkers in which the central acetylene group is replaced by a polar unit. 


\section{WILEY-VCH}

For the example shown in Figure 1 this is 1,3-difluorobenzene $(F)$; other polar linkers will be discussed below. Depending on the orientation of the dipolar elements (indicated by the brown arrows) and their arrangement one can create a number of different architectures of varying complexity. Two prototypical arrangements are the "stripe structure" and the "checkerboard structure" shown in Figure 1. We refrain from including functional groups containing reversible bonds like boronates or imines into the linkers of our model systems in order to keep the systems simple and not to divert from the main effects, even though in practice such elements are useful for achieving a high degree of crystallinity. ${ }^{[9]}$ As far as electrostatic design is concerned, their primary impact is an amplification or a reduction of the energetic shifts depending on whether their intrinsic dipoles are arranged parallel or antiparallel to those of the main polar units of the linkers.

In the third dimension, which is usually referred to as the stacking direction, we introduce vertical linkers that connect the zinc atoms of porphyrin units of subsequent layers (see Figure 1c). We specifically choose 1,4-diazobicycle[2.2.2]octane (DABCO) and 4,4'bipyridine as they are commonly used linkers for metal organic frameworks (MOFs). ${ }^{[30-33]}$ Moreover, in several instances they have been used to link zinc-porphyrin units. ${ }^{[34-36]}$

The band alignment in the above-described systems solely depends on the electrostatically triggered energetic shift between chemically identical semiconducting elements (the porphyrins), one can identify two major handles to tune the energetic offset: (i) one can either vary the magnitude of the dipole moment per in-plane linker by changing the polar group; (ii) alternatively, one can change the linker density. Notably, the two handles are not fully independent, as increasing the linker density reduces the dipole moment per linker due to depolarization effects. ${ }^{[10,32-34]}$ 

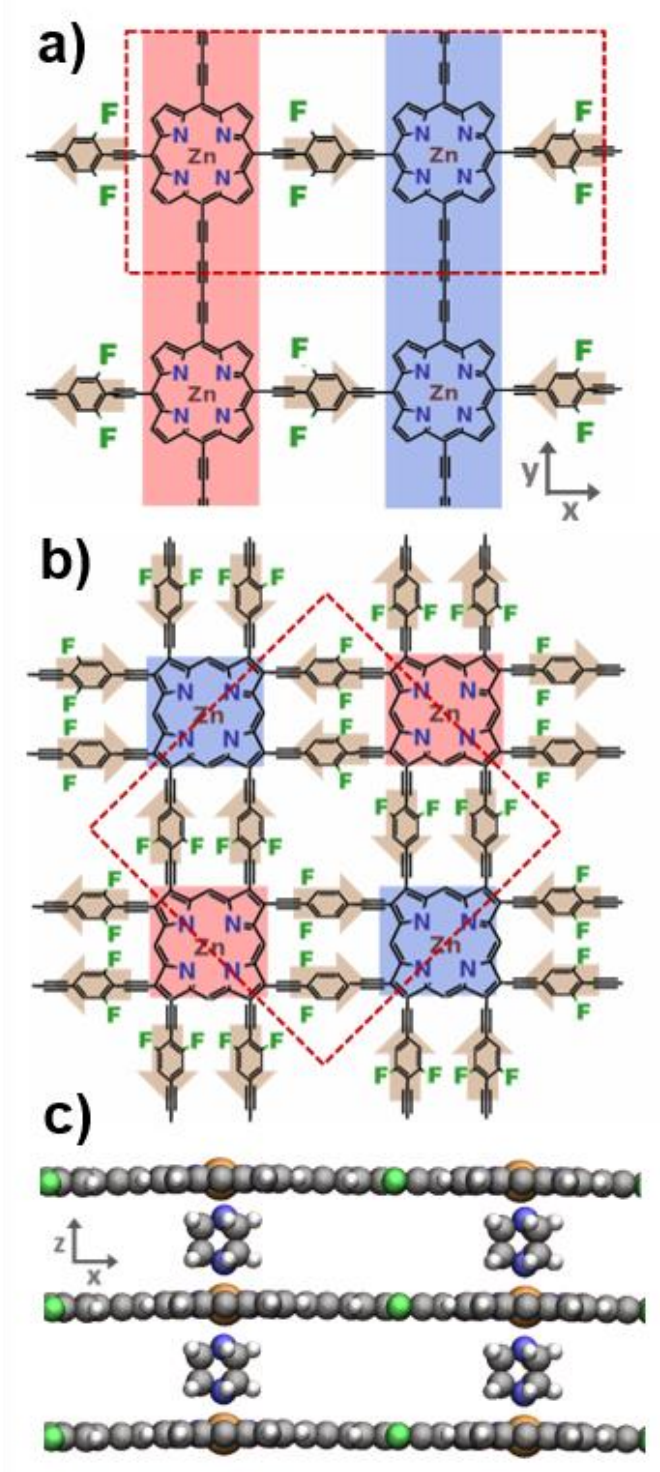

Figure 1: Chemical structures of prototypical examples of electrostatically designed covalent organic networks: (a) stripe architecture and (b) checkerboard structure. The red dashed lines indicate the unit cells used in the periodic DFT calculations. The arrows represent the directions of the dipole moments of the linking elements. The red (blue) shaded areas indicate regions where lowered (raised) electrostatic energies for electrons are expected. Besides the symmetry of the arrangement of the polar units the two displayed model systems also differ in their dipole densities. This is achieved by using singly (a) and doubly (b) linked structures (for more details see main text). A 3D representation of the model system is shown in (c), where 1,4-diazobicycle[2.2.2]octane (DABCO) represents a typical vertical linker. Color code for the atoms: grey $=C$, white $=H$, blue $=N$, orange $=Z n$, green $=F$. 


\section{WILEY-VCH}

To change the polarity of the linking groups we consider 1,3-dichlorobenzene $(C L)$, pyrimidine $(P Y R)$, 1,3-difluorobenzene $(F)$, and boron nitride $(B N)$. They exhibit dipole moments of $0.56,1.43,1.94$ and 6.86 Debye when calculated for an isolated singly linked porphyrin dimer. The differences between the various linkers are reduced when including them into the networks due to the above-mentioned depolarization effects, an effect that is fully accounted for in our periodic-boundary condition calculations.. The $B \equiv N$ linker is of hypothetical nature, but is still included here to show that very large shifts are feasible for large dipole moments. More realistic ways for achieving such large dipole moments would, for example, be multiple repetitions of the other polar elements.

The modification of the linker density can be achieved in two different ways: First, the distance in stacking direction can be changed by increasing the length of the vertical linker from DABCO to BIPYRIDINE. Additionally, varying between single- and double-linking of porphyrin units (cf., Figure $1 \mathrm{a}$ and $\mathrm{b}$ ) is a viable scenario considering that both linking motifs have been discussed in literature. ${ }^{[40-42]}$

To adopt a consistent nomenclature we introduce systematic abbreviations for the different model systems following the scheme [vertical linker]_[\#polar linkers]_[architecture]. In that spirit the material shown in Figure 1a is referred to as $D A B C O \_1 F \_$stripe and the one in Figure $1 \mathrm{~b}$ is denoted as $D A B C O \_2 F_{-}$checkerboard (in both cases assuming that DABCO linkers form the connection in the third dimension). The chemical structures of all systems can be found in Figure S5 (Supporting Information).

To present the general idea and discuss the underlying physics, we choose the $D A B C O \_1 F \_$stripe system as shown in Figure 1a. The corresponding contour plot of the electrostatic energy for an electron in a plane perpendicular to the stacking direction is shown in Figure 2. One clearly sees the expected division into stripes of high and low energy due to the superposition of the electric fields of the dipolar 1,3-difluorobenzene $(F)$ linkers. An interesting observation in this context is the relatively abrupt change of the energy, which is fully consistent with the work of Natan et al. ${ }^{[13]}$, who showed that the decay length of the field of a 2D array of point dipoles is nearly an order of magnitude shorter than the inter-dipole distance. 


\section{WILEY-VCH}

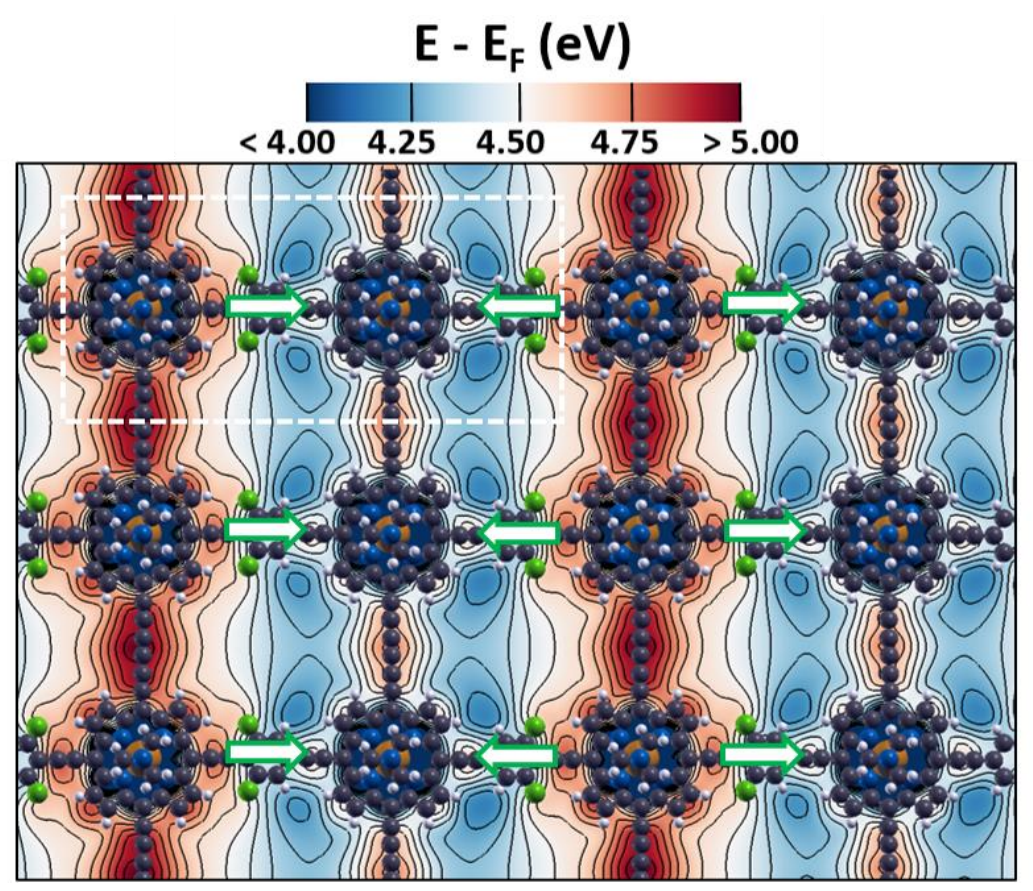

Figure 2. Contour plot of the electrostatic energy of an electron for the DABCO_lF_stripe system in a plane perpendicular to the stacking direction (approximately $2.5 \AA$ away from the molecular plane to avoid extensive variations near the nuclei). The electrostatic energy is given relative to the Fermi level. Isolines are drawn every $0.15 \mathrm{eV}$. The arrows represent the directions of the dipole moments of the linking elements. Color code for the atoms: grey $=C$, white $=H$, blue $=N$, orange $=Z n$, green $=F$.

To evaluate the consequences of these potential variations, it is useful to perform an in-depth analysis of the location of the electronic states in the 3D periodic system. In the absence of dipolar units, electronic states extend in both spatial directions due to the strong electronic coupling within the linked porphyrin plane. This is manifested in strongly dispersing bands in $\mathrm{X}$ - and Y-direction with band widths of $0.5 \mathrm{eV}$, see Figure S4 (Supporting Information). When introducing dipolar elements, this coupling is broken due to the electrostatically induced energetic shifts, diminishing the band width in the direction parallel to the dipoles to less than $0.1 \mathrm{eV}$. Hence, as can be seen from the schematic band alignment contained in Figure 3a, the electronic states should localize in the respective stripes and they should be energetically shifted with respect to each other. 

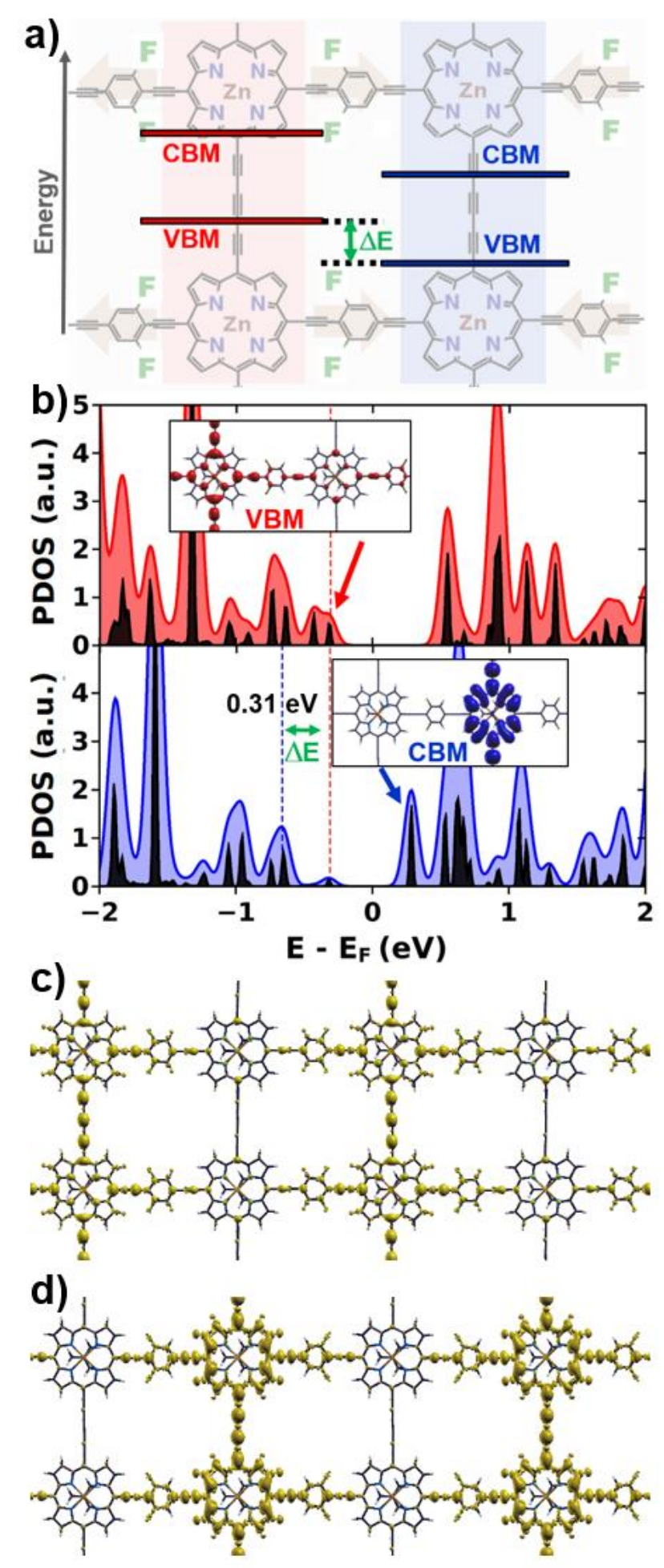

Figure 3. (a) Energy level alignment of the heterojunction achieved by electrostatic design for the DABCO_IF_stripe system. The band offset between VBMs (valence-band maxima) and CBMs (conduction-band minima) of electrostatically shifted stripes is indicated by $\triangle E$. (b) Density of states projected onto different parts of the unit cell for the DABCO_IF_stripe system; top panel: DOS projected onto the high electrostatic energy 


\section{WILEY-VCH}

side, i.e. red region in (a); bottom panel: DOS projected onto the low electrostatic energy side, i.e. blue region on (a). The energy is aligned to the Fermi level. The black lines represent the projected density of states (PDOS) obtained directly from the calculations with a Gaussian broadening of $0.01 \mathrm{eV}$ while the red and blue shaded areas show the PDOS with a Gaussian broadening of $0.05 \mathrm{eV}$ applied in a post-processing routine, serving as a guide to the eye. Isodensity plots of the Gamma-point states of the valence and conduction bands are included as insets (isovalue of 0.03 states per $\AA^{3}$ ). (c) and (d) show $3 D$ isodensity plots of the charge density differences between the neutral system and the equivalent positively $(c)$ and negatively $(d)$ charged DABCO_1F_stripe system. An isovalue of 0.0004 electrons per $\AA^{3}$ was used. More quantitative information independent of the choice of the isodensity value can be obtained by plotting the plane averaged charge density difference, see Figure S6 in the Supporting Information. There we also report the results of a Mulliken population analysis, which fully confirms the trends inferred from Figure $3 c$.

As shown in Figure 3b, these expectations are fully met in the present system, as can be inferred from the densities of states (DOS) projected onto regions with different electrostatic energy (the regions shaded in blue and red in Figure 3a). One observes an essentially rigid shift of the projected DOSs amounting to $0.31 \mathrm{eV}$. This shift represents the band offset between the local valence-band maxima VBM, respectively, conduction-band minima CBM of two consecutive stripes (cf., Figure 3a). As the stripes are chemically identical, this offset is solely determined by the fields arising from the polar 1,3difluorobenzene $(F)$ linking groups. In the Supporting Information we included a section highlighting the collectivity of the effect and showing that mesomeric effects caused by the fluorine atoms do not significantly impact the situation. The fact that the frontier bands below and above the Fermi level are localized in different spatial regions can be clearly seen from the corresponding local densities of states (LDOSs) plotted as insets in Figure $3 \mathrm{~b}$. This suggests that in these electrostatically designed covalent networks, pathways for electrons and holes should be decoupled from each other.

To verify that, we studied the localization of excess electrons and holes in the system by either adding or removing 0.1 electrons per unit cell (containing two porphyrin units). Such local excess charges could result either from exciton dissociation or from carrier 


\section{WILEY-VCH}

injection at electrodes. The charge density differences between the neutral and charged systems are shown in Figure $3 \mathrm{c}$ and $\mathrm{d}$. They nicely correlate with the isodensity plots of the LDOSs of the frontier states (insets of Figure 3b). This is insofar remarkable, as the localization of the charges occurs without considering the strong electron-phonon coupling in organic semiconductors (often also termed polaronic effects), which usually drive spatial localization of charge carriers in such materials.

Exciton binding energies in organic semiconducting materials have been reported to range from a few tenths of an $\mathrm{eV}$ up to several $\mathrm{eV} .{ }^{[43-45]}$ As a driving force for dissociating these excitons into separate charge carriers, one usually exploits the offset of the energy levels in type-II bulk heterojunctions. Consequently, this offset needs to be sufficient for ensuring efficient exciton dissociation. At the same time, it should not be too large as this would result in an unnecessary dissipation of energy and, thus, in a reduction of the achievable open-circuit voltage, when using the materials in solar cells. In this context, a significant advantage of the electrostatic design strategy over changing the chemical nature of the semiconducting units is that it offers a comparably straightforward way of quasi continuously tuning the shift of the energy levels in the nominally electron accepting and donating sections. As mentioned already above, this can be achieved by tuning the dipole density either through varying the dipole moment of the linkers, the number of linkers between porphyrin units, or the spacing between the layers. As shown in Figure 4, in this way we are indeed capable of significantly varying the band-offset for the considered systems.

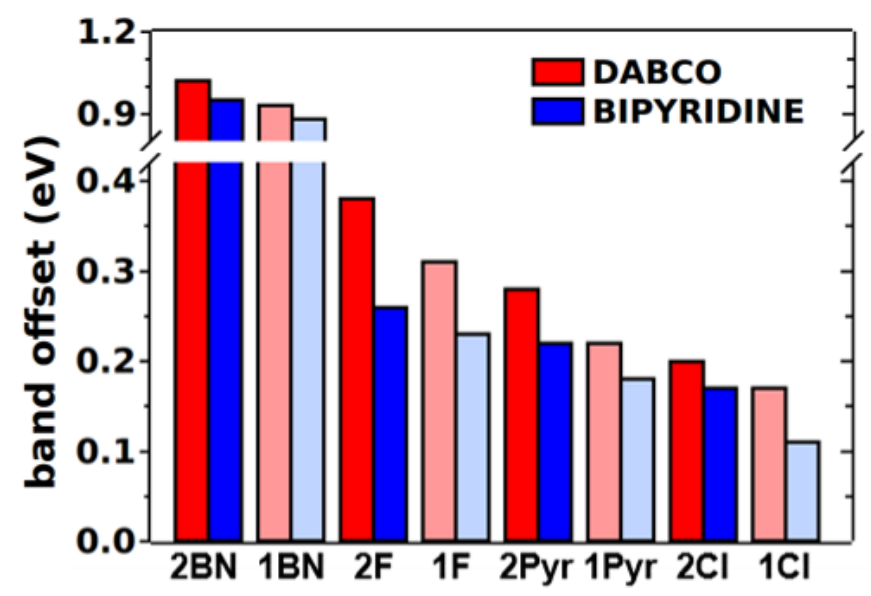

Figure 4. Band offset, $\triangle E$, between VBMs and CBMs of electrostatically different stripes achieved by collective electrostatic design. $\Delta E$ can be quasi continuously tuned between 


\section{WILEY-VCH}

0.11 to $1.02 \mathrm{eV}$ depending on the used dipolar elements and linker densities. The used polar linkers are 1,3-dichlorobenzene $(C L)$, pyrimidine $(P Y R)$, 1,3-difluorobenzene $(F)$, and boron nitride (BN). Pale bars refer to singly-linked structures, while solid bars refer to the doubly-linked equivalents. The chemical structures of all systems can be found in Figure S5 (Supporting Information). The values for $\triangle E$ are taken from the PDOS plots shown in Figure S7-S10 and summarized in Table S2 in the Supporting Information.

Electrostatic design also allows the realization of a multitude of complex quantum structures, where the checkerboard architecture introduced in Figure 1b is only one example. The corresponding contour plot of the electrostatic energy is shown in Figure 5a. The obtained band offset ( $0.47 \mathrm{eV}$ for $D A B C O \_2 F_{-}$checkerboard $)$and the projected densities of states are similar to the stripe structure, but here, carrier transport within the sheets is largely suppressed. This can be gauged from the observation that the band-dispersion essentially vanishes both in the $\mathrm{X}$ and $\mathrm{Y}$-directions. I.e., compared to the striped structure, now also the band width in Y-direction is reduced from $0.15(0.35) \mathrm{eV}$ to $0.00(0.15) \mathrm{eV}$ for the valence and conduction bands, respectively (see Figure S11 in the Supporting Information).

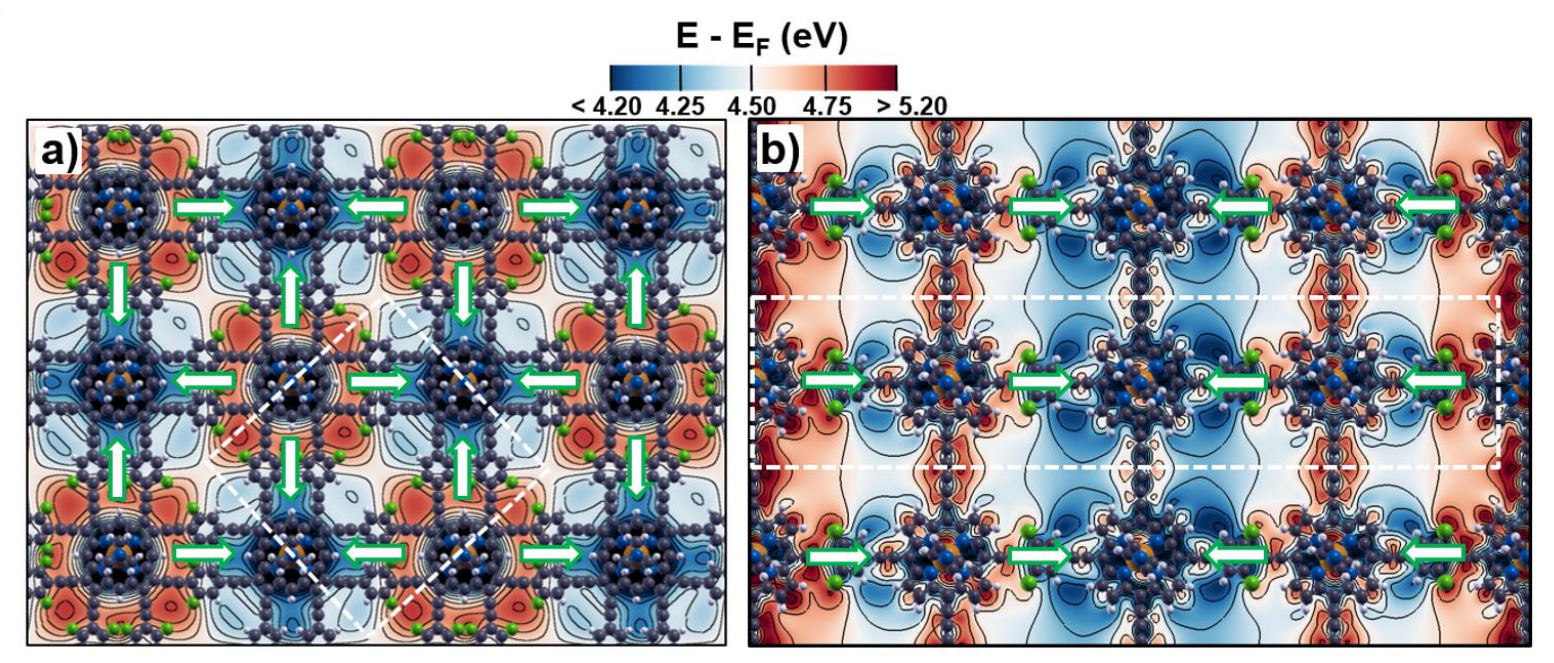

Figure 5. Contour plot of the electrostatic energy for $(\boldsymbol{a})$ the DABCO_2F_checkerboard system and (b) the BIPYRIDINE_IF_cascade system in a plane perpendicular to the stacking direction, approximately $2.5 \AA$ away from the molecular plane to avoid extensive variations in the energy near the nuclei. The energy is given relative to the respective Fermi level. Isolines 


\section{WILEY-VCH}

are drawn every $0.15 \mathrm{eV}$. Unit cells are indicated as dashed white boxes. The arrows represent the directions of the dipole moments of the linking elements. Color code for the atoms: grey $=C$, white $=H$, blue $=N$, orange $=Z n$, green $=F$.

Another interesting structure that is much more difficult to realize with conventional strategies is the quantum cascade architecture, which is shown in Figure $5 b$ for the example of BIPYRIDINE_lF_cascade (the corresponding chemical structure is shown in Figure S12, Supporting Information). Compared to the stripe topology discussed above, where the dipoles in the linking groups connecting successive stripes point in opposite directions, here the dipole orientation of the linkers switches only after several (in the displayed system after two) stripes. This creates an energetic staircase, as can again be inferred from the electrostatic energy shown in Figure 5b. The electronic states follow the characteristics of the energy landscape resulting in a subsequent shift of about $0.3 \mathrm{eV}$ between the band edges in subsequent regions (see Figure S13, Supporting Information). In this way, the highest and lowest energy stripes (and consequently also the states at the corresponding band edges) are well separated in space. Such a structure could, for example, help minimizing recombination losses in photovoltaic cells.

In conclusion, based on first-principle calculations, we have shown that electrostatic design is a highly promising approach for manipulating the electronic properties of bulk materials. To illustrate how charges can be controlled at the nanoscale through ordered arrangements of polar units, we studied 3D covalent organic networks and discussed their properties primarily in view of their potential applications in photovoltaics. Through electrostatic design we achieve an energy-level offset between otherwise chemically identical semiconducting segments. This is in stark contrast to conventional donor-acceptor COFs, where chemically distinct building blocks provide the donor and acceptor functionality needed to dissociate excitons. Through the inclusion of polar building blocks into the networks, we are able to manipulate the electronic landscape in a controlled way, achieving spatially confined pathways for electrons and holes. Varying the dipole density in the considered systems offers the possibility to continuously tune the magnitude of the band offset over a wide energy range. Changing the position and orientation of the introduced dipolar elements allows the design of architectures with conceptually different potential distributions, such as stripes, checkerboards or quantum-cascades. These aspects endow the 
electrostatic design approach with significant potential for the realization of materials with novel properties far beyond the organic networks discussed here.

\section{Experimental Section}

To determine the electronic structure of the systems and optimize their geometries, we performed band-structure calculations within the framework of density-functional theory (DFT) using the Fritz-Haber-Institute ab initio molecular simulations (FHI-aims) code. ${ }^{[46]}$ We applied the Perdew-Burke-Ernzerhof $(\mathrm{PBE})^{[47]}$ exchange-correlation functional augmented with the Tkatchenko-Scheffler scheme ${ }^{[48]}$ to account for van der Waals interactions for geometry optimization and the hybrid functional HSE06 ${ }^{[49]}$ for single point calculations. We applied a converged set of light basis functions at the tier2 level and a $2 \times 4$ x 20 Monkhorst-Pack k-point grid for all studied systems in stripe architecture. For the checkerboard structure we used a converged k-point grid of $4 \times 4 \times 20$. Full details on the applied computational methodology (in particular also the pursued geometry optimization procedure) and numerical parameters used in our calculations are contained in the Supporting Information. The latter also contains a discussion on how the electronic structure of the materials is changed when employing the PBE (i.e. a generalized gradient approximation based functional) instead of the hybrid HSE06 functional. VMD ${ }^{[50]}, \mathrm{XCrySDen}^{[51]}$ and Mayavi2 ${ }^{[52]}$ were used for graphical visualization.

\section{Supporting Information}

Supporting Information is available from the Wiley Online Library or from the author.

\section{Acknowledgements}

We thank Christian Slugovc, and Christoph Wöll for stimulating discussions. Financial support by the Austrian Science Fund (FWF): P28051-N36 is gratefully acknowledged. The computational studies presented have been achieved using the Vienna Scientific Cluster (VSC). 


\section{WILEY-VCH}

Received: ((will be filled in by the editorial staff))

Revised: ((will be filled in by the editorial staff)) Published online: ((will be filled in by the editorial staff))

\section{References}

[1] X. Feng, L. Chen, Y. Honsho, O. Saengsawang, L. Liu, L. Wang, A. Saeki, S. Irle, S. Seki, Y. Dong, D. Jiang, Adv. Mater. 2012, 24, 3026.

[2] M. Dogru, M. Handloser, F. Auras, T. Kunz, D. Medina, A. Hartschuh, P. Knochel, T. Bein, Angew. Chem. 2013, 125, 2992.

[3] S. Jin, X. Ding, X. Feng, M. Supur, K. Furukawa, S. Takahashi, M. Addicoat, M. E. ElKhouly, T. Nakamura, S. Irle, S. Fukuzumi, A. Nagai, D. Jiang, Angew. Chem. Int. Ed. 2013, 52, 2017.

[4] S. Jin, K. Furukawa, M. Addicoat, L. Chen, S. Takahashi, S. Irle, T. Nakamura, D. Jiang, Chem. Sci. 2013, 4, 4505.

[5] M. Dogru, T. Bein, Chem Commun 2014, 50, 5531.

[6] L. Chen, K. Furukawa, J. Gao, A. Nagai, T. Nakamura, Y. Dong, D. Jiang, J. Am. Chem. Soc. 2014, 136, 9806.

[7] M. Calik, F. Auras, L. M. Salonen, K. Bader, I. Grill, M. Handloser, D. D. Medina, M. Dogru, F. Löbermann, D. Trauner, A. Hartschuh, T. Bein, J. Am. Chem. Soc. 2014, 136, 17802.

[8] S. Jin, M. Supur, M. Addicoat, K. Furukawa, L. Chen, T. Nakamura, S. Fukuzumi, S. Irle, D. Jiang, J. Am. Chem. Soc. 2015, 137, 7817.

[9] N. Huang, P. Wang, D. Jiang, Nat. Rev. Mater. 2016, 1, 16068.

[10] S.-Y. Ding, W. Wang, Chem Soc Rev 2013, 42, 548.

[11] D. Cahen, R. Naaman, Z. Vager, Adv. Funct. Mater. 2005, 15, 1571.

[12] A. Natan, Y. Zidon, Y. Shapira, L. Kronik, Phys. Rev. B 2006, 73, 193310.

[13] A. Natan, L. Kronik, H. Haick, R. T. Tung, Adv. Mater. 2007, 19, 4103.

[14] G. Heimel, F. Rissner, E. Zojer, Adv. Mater. 2010, 22, 2494.

[15] O. L. A. Monti, J. Phys. Chem. Lett. 2012, 3, 2342.

[16] T. Abu-Husein, S. Schuster, D. A. Egger, M. Kind, T. Santowski, A. Wiesner, R. Chiechi, E. Zojer, A. Terfort, M. Zharnikov, Adv. Funct. Mater. 2015, 25, 3943.

[17] G. J. Kraberger, D. A. Egger, E. Zojer, Adv. Mater. Interfaces 2015, 2, n/a.

[18] B. Kretz, D. A. Egger, E. Zojer, Adv. Sci. 2015, 2, n/a.

[19] A. Kovalchuk, T. Abu-Husein, D. Fracasso, D. A. Egger, E. Zojer, M. Zharnikov, A. Terfort, R. C. Chiechi, Chem. Sci. 2016, 7, 781.

[20] E.-Y. Choi, P. M. Barron, R. W. Novotny, H.-T. Son, C. Hu, W. Choe, Inorg. Chem. 2009, 48, 426.

[21] A. Nagai, X. Chen, X. Feng, X. Ding, Z. Guo, D. Jiang, Angew. Chem. Int. Ed. 2013, 52, 3770.

[22] X. Ding, X. Feng, A. Saeki, S. Seki, A. Nagai, D. Jiang, Chem. Commun. 2012, 48, 8952. 


\section{WILEY-VCH}

[23] B. J. Burnett, P. M. Barron, W. Choe, CrystEngComm 2012, 14, 3839.

[24] M. C. So, S. Jin, H.-J. Son, G. P. Wiederrecht, O. K. Farha, J. T. Hupp, J. Am. Chem. Soc. 2013, 135, 15698.

[25] N. Huang, X. Chen, R. Krishna, D. Jiang, Angew. Chem. 2015, 127, 3029.

[26] X. Chen, J. Gao, D. Jiang, Chem. Lett. 2015, 44, 1257.

[27] H. Wang, H. Ding, X. Meng, C. Wang, Chin. Chem. Lett. 2016, 27, 1376.

[28] C. W. Tang, Appl. Phys. Lett. 1986, 48, 183.

[29] M. G. Walter, A. B. Rudine, C. C. Wamser, J. Porphyr. Phthalocyanines 2010, 14, 759.

[30] J. Kesters, P. Verstappen, M. Kelchtermans, L. Lutsen, D. Vanderzande, W. Maes, Adv. Energy Mater. 2015, 5, 1500218.

[31] O. Shekhah, H. Wang, M. Paradinas, C. Ocal, B. Schüpbach, A. Terfort, D. Zacher, R. A. Fischer, C. Wöll, Nat. Mater. 2009, 8, 481.

[32] J. Zhuang, J. Friedel, A. Terfort, Beilstein J. Nanotechnol. 2012, 3, 570.

[33] B. Liu, O. Shekhah, H. K. Arslan, J. Liu, C. Wöll, R. A. Fischer, Angew. Chem. Int. Ed. 2012, 51, 807.

[34] Z. Wang, J. Liu, H. K. Arslan, S. Grosjean, T. Hagendorn, H. Gliemann, S. Bräse, C. Wöll, Langmuir 2013, 29, 15958.

[35] P. N. Taylor, H. L. Anderson, J. Am. Chem. Soc. 1999, 121, 11538.

[36] C. A. Hunter, M. N. Meah, J. K. M. Sanders, J. Am. Chem. Soc. 1990, 112, 5773.

[37] E. Iengo, E. Zangrando, R. Minatel, E. Alessio, J. Am. Chem. Soc. 2002, 124, 1003.

[38] D. Cornil, Y. Olivier, V. Geskin, J. Cornil, Adv. Funct. Mater. 2007, 17, 1143.

[39] D. Deutsch, A. Natan, Y. Shapira, L. Kronik, J. Am. Chem. Soc. 2007, 129, 2989.

[40] L. Romaner, G. Heimel, E. Zojer, Phys. Rev. B 2008, 77, 045113.

[41] P. N. Taylor, J. Huuskonen, R. T. Aplin, H. L. Anderson, J. Huuskonen, G. Rumbles, E. Williams, Chem. Commun. 1998, 909.

[42] I. Hisaki, S. Hiroto, K. S. Kim, S. B. Noh, D. Kim, H. Shinokubo, A. Osuka, Angew. Chem. Int. Ed. 2007, 46, 5125.

[43] T. Tanaka, A. Osuka, Chem Soc Rev 2015, 44, 943.

[44] M. Knupfer, Appl. Phys. A 2003, 77, 623.

[45] A. Dkhissi, Synth. Met. 2011, 161, 1441.

[46] P. K. Nayak, Synth. Met. 2013, 174, 42.

[47] V. Blum, R. Gehrke, F. Hanke, P. Havu, V. Havu, X. Ren, K. Reuter, M. Scheffler, Comput. Phys. Commun. 2009, 180, 2175.

[48] J. P. Perdew, K. Burke, M. Ernzerhof, Phys. Rev. Lett. 1996, 77, 3865.

[49] A. Tkatchenko, M. Scheffler, Phys. Rev. Lett. 2009, 102, 073005.

[50] J. Heyd, G. E. Scuseria, M. Ernzerhof, J. Chem. Phys. 2003, 118, 8207.

[51] W. Humphrey, A. Dalke, K. Schulten, J. Mol. Graph. 1996, 14, 33.

[52] A. Kokalj, J. Mol. Graph. Model. 1999, 17, 176.

[53] P. Ramachandran, G. Varoquaux, Comput. Sci. Eng. 2011, 13, 40. 


\section{WILEY-VCH}

The electrostatic design of $3 \mathrm{D}$ covalent organic networks is presented as an innovative strategy to mimic donor-acceptor bulk heterojunctions. Periodic arrangements of polar building blocks are used to locally shift the energies of the electronic states and to spatially confine the frontier wavefunctions. In this way a driving force for exciton dissociation is realized without the need for using distinct semiconducting units.

Keywords: covalent organic frameworks, electrostatic design, solar cells, density functional theory

V. Obersteiner, A. Jeindl, J. Götz, A. Perveaux, Oliver T. Hofmann, and E. Zojer*

\section{Electrostatic Design of 3D Covalent Organic Networks}

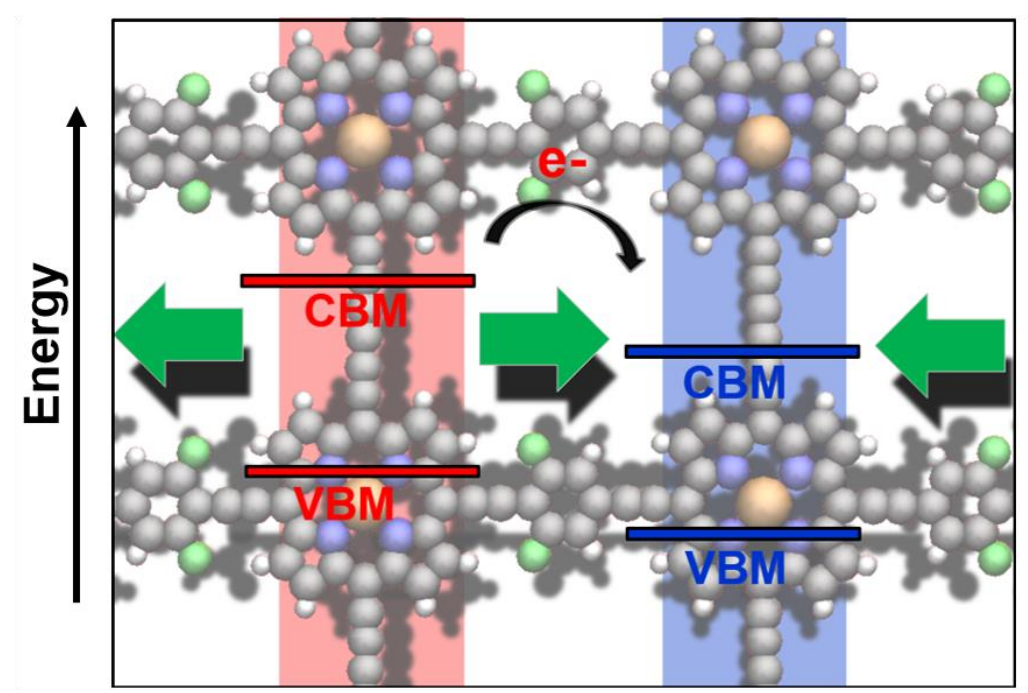

